\title{
Influence of temporal parameters of laser irradiation on emission spectra of the evaporated material
}

\author{
E. Zabello, V. Syaber, A. Khizhnyak \\ International Center «Institute of Applied Optics» of National Academy of Sciences of Ukraine,_254053, Kyiv, Ukraine, \\ phone: (38044) 212-21-58; fax: (38044) 212-48-12khizh@writeme.com
}

\begin{abstract}
Adstract. The results of study of the emission spectra of metals excited by laser pulses bursts under atmospheric conditions are presented. It is demonstrated that the area responsible for the atom irradiation of evaporated substance is essentially distant from that of the continual spectrum irradiation. As a result, the sensitivity of the qualitative analysis increases. For quantitative analysis it is necessary to select the analytical lines differing from those used for spark or arc emission analysis.
\end{abstract}

Keywords: laser, emission spectrum, erosive torch.

Paper received 16.02.99; revised manuscript received 12.05.99; accepted for publication 24.05.99.

\section{Introduction}

Despite evident advantages of the laser emission spectroscopy (LES) [1] the method did not find yet wide practical application. The primary problem here is low detection ability, i.e., high threshold level in detecting the low concentrated elements in a sample. This low detection sensitivity of the LES technique is basically caused by high intensity continuos spectra that comes along with discrete spectra whose components are associated with material under the study and therefore of primary interest.

An improvement of the laser-induced emission spectra characteristics through complementary electrical discharge of the evaporated media [2] substantially complicates the whole system and adds limitations immanent to this type of excitation. Emission spectra of much better quality can be also achieved by reducing atmospheric pressure around a sample under test [3], however this will require vacuum chamber, which complicates the entire system.

The process of the emitted media torch formation is entirely non-stationary and can be divided onto several consequent stages. First, it starts with absorption of the laser radiation by a target under investigation with its local overheating above melting temperature. This leads to an explosive outbreak of the object's substance, flash of plasma, breakdown and overheating of the surrounding air layer, and finally to emission of continuos spectra. The formed cloud of vapor enlarges and produces a shock wave. This moving shock wave leaves behind itself an area with reduced pressure. Then as the temperature of the expanding cloud of vapor decreases, the atoms of the evaporated substance are getting down to lower levels, the ions recombine, and quasi-steady thermodynamic equilibrium is established. However, the vapor of the substance formed after a single pulse action can't move away from the target's surface, being resisted by the pressure of the surrounding atmosphere. So the area of luminous vapor responsible for linear spectrum is overlapped with the area of continuous spectrum, which decreases the detection limit of laser emission method.

It was demonstrated in [4-6] that a significant transformation of the laser induced torch radiation is observed when two laser pulses (with ten microsecond interval) 


\section{E. Zabello et al.: Influence of temporal parameters of ...}

expose the surface, as being compared with single pulse action. Basing on those results and taking into account the complicated multi-step mechanism of the formation of excited atoms, it is interesting to learn the influence of temporal characteristics of laser radiation on a character of erosive torch radiation, when the initiating laser is composed by a train of the pulses. The results of such investigations are presented below.

\section{Experimental set-up}

A Q-switched YAG: $\mathrm{Nd}^{3+}$ laser with two different $\mathrm{LiF}$ crystals (initial transparency $T_{01}=70 \%$ and $T_{02}=90 \%$, respectively) as passive Q-modulator was used in these experiments. Placing the crystal into laser cavity resulted in formation of the train of pulses with an interval $10 \mu$ s or $15 \mu$ s respectively. The $40 \mu$ s time delay was observed when both crystals were inserted simultaneously. The output energy 10, 15 and $20 \mathrm{~mJ}$ was obtained at pumping energy $75 \mathrm{~J}$ and flash lamp time $200 \mu \mathrm{s}$. The pumping energy variation affected both the generated pulses number and the interval in their sequence, leaving the output energy of the every pulse unchanged. The laser radiation was focused into the target's surface by lens with $F=75 \mathrm{~mm}$ in the spot $100 \mu \mathrm{m}$ diameter. The studied samples of copper, iron and tin, as well as their alloys were used for analysis. Such selection was made because of difference in thermo-physical properties, which allows to expand the obtained results onto some wider class of materials.

Detection of the luminescent spectra was made with spectrograph DFS-452 (on photographic film) or linear CCD-array. By using the film, we could receive additional information about change of torch radiation along of its axis. The CCD-array in its turn made it possible to achieve direct PC data processing. However, because of relatively small dimension of the array only limited narrow spectral band (about $10 \mathrm{~nm}$ ) was taken.

\section{Experimental results}

Performed measurements revealed an essential dependence of the emission spectrum behavior upon temporal characteristics of the laser pulses. The most striking changes have been observed with first type of the Qmodulator $\left(T_{01}\right)$. In this case, the maximum range of reproducability for a quantity of pulses and interpulse separations could be achieved. By varying the pumping energy from threshold level $15 \mathrm{~J}$ up to $75 \mathrm{~J}$, the number of the pulses in train was changed from 1 to $18-20$, while interpulse separation smoothly decreased from $20 \mu$ s (two pulses) down to $10 \mu \mathrm{s}$.

Fig. 1 illustrates the character of the emission spectra for the steel sample DM-2 which is composed by $98 \%$ of iron. Let's admit that all these spectra were obtained at equal exposure level of the sample. This means that the total amount of energy affecting the sample was managed to remain the same, i.e., the variation of the

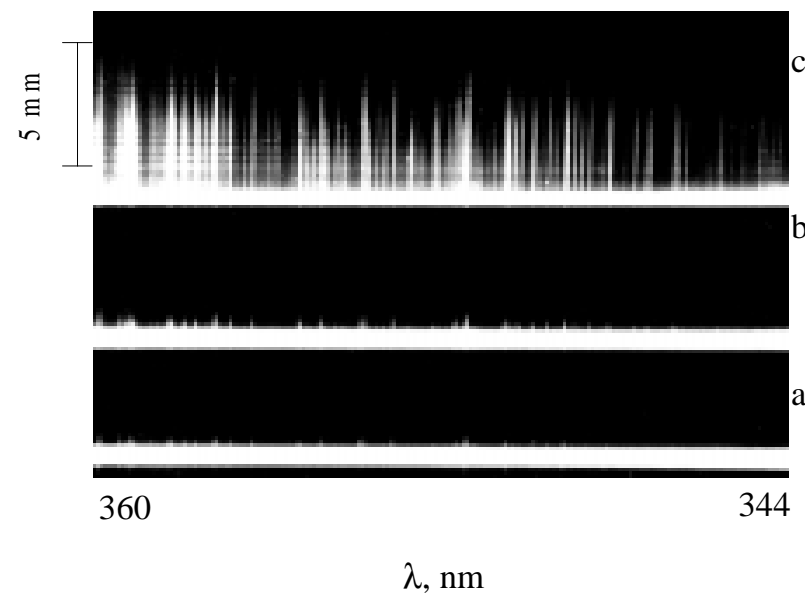

Fig. 1. The emission spectrum photo of steel sample for different number of laser pulses: $\mathrm{a}-$ monopulses, $\mathrm{b}$ - double pulses, $\mathrm{c}-18-20$ pulses in packet. Vertical coordinate is distance along the input aperture of spectrograph.

number of pulses was compensated by corresponding variation of their energy.

For single $10 \mathrm{~mJ}$ pulse of $10 \mathrm{~ns}$ (Fig. 1a) an intensive continuous background was observed within all spectral range, being completely overlapped with entire range of the discrete spectra.

In double-pulse mode (pulse separation $20 \mu \mathrm{s}, 10 \mathrm{~mJ}$ in each pulse) certain increase of the discrete spectrum radiation intensity took place at the torch's top, with slightly narrowed range of continuous radiation (Fig. 1b). As the pumping level increases (which is accompanied by concurrent increase in the number of pulses in a train and decrease in their time interval) a certain increase of the discrete spectrum range takes place with simultaneous narrowing of a continuous radiation component. A lasting regime with $18-10$ pulses in train and $10 \mu$ s separations was found to be optimal (Fig. 1c). The results of measurements of the torch's height as a function of the number of pulses in a train are shown in the Table 1.

As follows from the above results, the action of the train of pulses on the target leads to a significantly increase of the torch's area that emits the discrete spectrum. An area of radiation of continuous spectrum decreases. Therefore the area of the torch available for caring out spectral analysis decreases. Moreover, in this case we observed strong increase in the number of the spectral lines of the discrete spectrum as their luminous zone is beyond that of the continuous spectrum. Thus, the analytical spectrum quality and detecting limit increase.

When crystal number 2 or combination of both crystals was used, qualitatively similar changes of spectrum were observed. However, in this case both the intensity of the discrete spectrum and the ratio between the width of «discrete zone» and the corresponding width of «continual zone» became worse. 
E. Zabello et al.: Influence of temporal parameters of ...

Table 1. Dependence of torch height on number of pulses in train for copper and steel target.

\begin{tabular}{cccc}
\hline Conditions & Material & Torch height, $\mathrm{mm}$ & Background height, $\mathrm{mm}$ \\
\hline \multirow{2}{*}{ 1 pulse } & $\mathrm{Cu}$ & 1,15 & 0,85 \\
& $\mathrm{Fe}$ & 1,10 & 0,80 \\
2 pulses & $\mathrm{Cu}$ & 1,30 & 0,85 \\
& $\mathrm{Fe}$ & 1,80 & 0,80 \\
7 pulses & $\mathrm{Cu}$ & 3,20 & 0,45 \\
& $\mathrm{Fe}$ & 4,50 & 0,35 \\
& $\mathrm{Cu}$ & 4,30 & 0,35 \\
& $\mathrm{Fe}$ & 6,40 & 0,25 \\
\hline \hline
\end{tabular}

Measurements of the spectral line intensities $(I)$ were made with a CCD-array. The spectrograms for the sample of steel for three above described cases are presented in Fig. 2. The measurements were performed for zone of the torch lying above the area of continual radiation. With increasing the number of pulses in the train and decreasing their interval, the growth of the intensity of the weak lines is observed. Such an enrichment of the detected spectra at the expense of the weak lines increases sensitivity for both qualitative and quantitative analysis.

The spectrograms corresponding to different zones of the torch are presented in Fig. 3. Here, the plot a (Fig. 3a) matches to the lower zone of the torch (but higher than zone with continual spectrum). Figs $3 b$ and $3 c$ belong to the central and upper torch's zones respectively.

Apparently, the ion's associated lines as well as slander background have been observed in the area of the torch that is close to the target's surface. With increasing distance from the surface, because of the ion recombination, the whole spectra acquire the character typical for atoms which simplifies its interpretation. Furthermore, with transition to distant regions of the torch the line width in the emission decreases (Fig. 4), which indicates the temperature decrease in this area.

It follows from the performed measurements of the integral torch luminescence that its duration is about $1 \mu \mathrm{s}$, which is considerably shorter than an interval between the laser pulses themselves. When this interval was selected to be longer than $20 \mu \mathrm{s}$, the ratio between the amplitude of the torchluminescence and the amplitude of corresponding laser pulses remains unchanged within the limit of all train. By reducing the time interval in the train to about $10 \mu$ s this ratio became dependent upon the number of pulse, reaching its maximal value for the every third pulse.

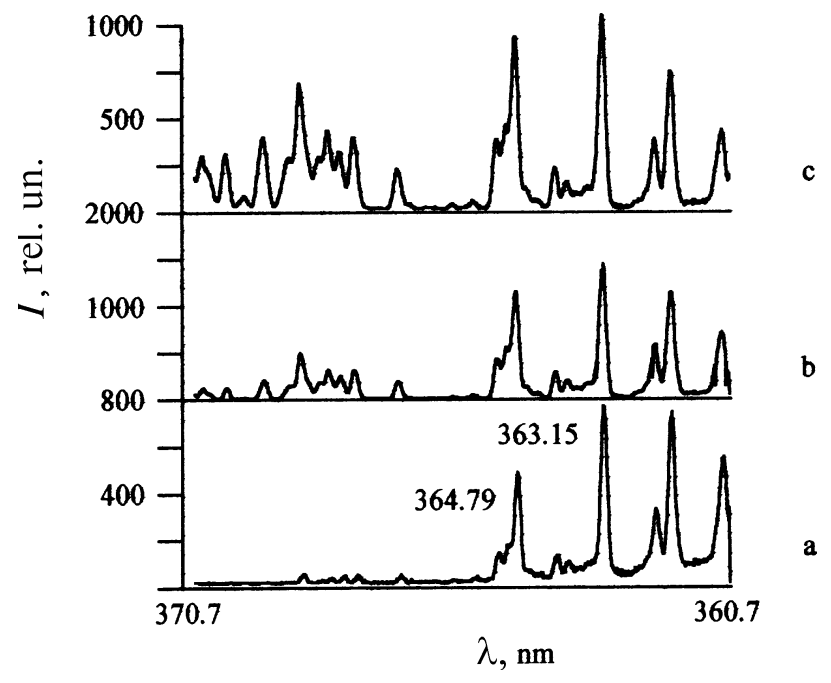

Fig. 2. Emission spectrum of steel sample: $a-$ monopulses, $b-$ double pulses, $\mathrm{c}-18-20$ pulses in packet.

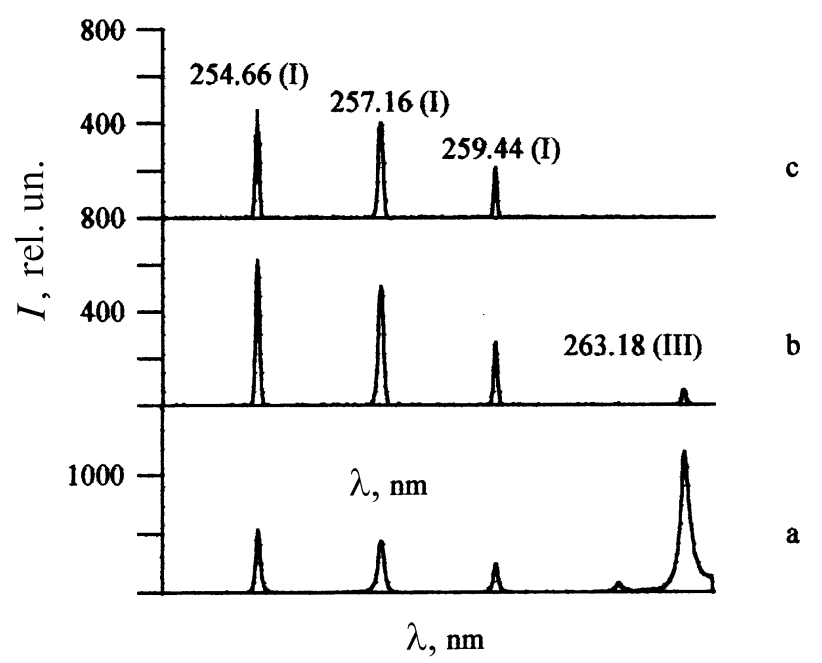

Fig. 3. Emission spectrum of tin sample for different parts of torch: a - lower zone, $\mathrm{b}$ - middle zone, $\mathrm{c}$ - upper zone. 


\section{E. Zabello et al.: Influence of temporal parameters of ...}

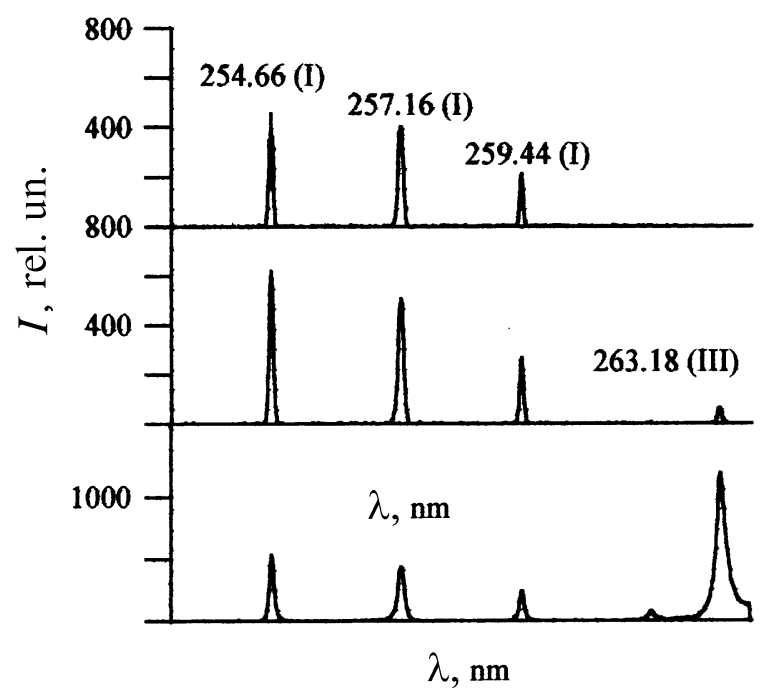

Fig. 4. The contour of line $\mathrm{Zn} 255.796$ (II): 1 - upper zone of torch, 2 - lower zone of torch.

\section{Discussion}

According to $[4,5]$, where the double-pulse action was studied, the observed improvement in the signal-to-noise ratio can be explained by formation of zone with low pressure around the interaction area. Therefore the second laser pulse takes place under lower pressure conditions.

However, the effects detected in our experiments can not be explained by only this factor [7]. Indeed, the component with continuous emission spectra can be reduced with the atmospheric pressure decrease. Moreover, the vapors ejected by subsequent pulse will propagate in the area with lower pressure, whicht makes it possible for this vapor to keep its kinetic energy and to shift the shell of the atmosphere away on a greater distance. This can explain an enlargement of the torch (see Tab. 1).

Moreover, every consequent pulse ejects the vapor into the area that contains the evaporated substance generated by previous pulses. As a result, the propagating front of the new portion of the evaporated substance affects and interacts not only with pure air, like the first pulse does, but with the vapor already containing the target's substance. All that makes the excitation process to be more effective and in this way enhances the intensity of the signal.

Finally, as the ejected vapors propagate in a space with decreased pressure, they can move on larger distance and move away from the target's surface, in other words, leave the area where the continuous spectrum is formed. Due to larger propagation length the ions have enough time for their recombination, while the atoms have not time for deactivation, so they drop to quasisteadystate condition. This last process is accompanied by discrete spectra emission as it is seen from the abovepresented picture.

Apparently, when the interval between the pulses in train coincide with time of the formation of maximum concentration of vapor in the torch area [8], the conditions of excitation becomes nearly optimal. In our exberiments such regime corresponds to $10 \mu$ s interval between the pulses.

Thus, in going to multiple pulse laser regime a significant enlargement of the radiating zone of the torch takes place, and, as a result, the number of registered lines increases. By using such form of multiple pulse action the achieved detection limit was about $10 \mathrm{ppm}$ at standard atmospheric conditions without any additional means of excitation.

The potentials of quantitative analysis were investigated on the samples of steel standards 461-464, one of which being used for calibration. The paires of the lines analogous to those for quantitative analysis with spark discharge method were selected in these experiments. For a concentration of the impurities not less than $10 \%$, the error of measurements was about $2 \%$, and at impurity levels less than $1 \%$ the error reached $50 \%$. The disproportional changes in correlation between the intensities of the spectral lines for the material with strongly different concentration of components are responsible for this result. The comparison of spectra obtained at laser excitation and those at spark discharge shows their radical difference, for both the number of the registered lines and their relative intensities. That is why, performing the analysis with LAS technique, there is a strong necessity in selection of the additional analytical lines, as well as the most optimal spectral range for such measurements.

\section{Acknowledgement}

Authors wish to express their thanks to Dr. S. Anokhov of the International Center "Institute of Applied Optics" for fruitful discussions contributed much to the obtaining results.

\section{References}

1. W. W. Duley, Laser Processing and Analysis of Materials, Plenum Press, New York and London (1983).

2. S. V. Oshemkov, A.A. Petrov, Spectrum analysis with laser atomization // Zhurnal prikladnoi spekrtoskopii, 48(3), pp.359-376, (1986) (in Russian).

3. V. A. Rozantsev, M. L. Petuh, A. A. Yankovsky, Influence of air pressure on spectra of laser plasma // Zhurnal prikladnoi spekrtoskopii, 47(4), pp.549-553, (1987) (in Russian).

4. A. Y. Buharov, S. M. Pershin, Changing of laser plasma parameters when turning to double-pulse irradiating of dielectric in air / / Zhurnal prikladnoi spekrtoskopii, 51(4), pp.564-571, (1989) (in Russian).

5. M. L. Petuh, A. D. Shirokanov, A. A. Yankovsky, The investigation of plasma spectra formed by paired laser pulses // Zhurnal prikladnoi spekrtoskopii, 61(5-6), pp.340-344, (1994) (in Russian). 
E. Zabello et al.: Influence of temporal parameters of ...

6. 6. R. Satman, V. Sturm, R. Noll, Laser-induced break down spectroscopy of steel samples using multiple Q-switch Nd: YAG laser pulses // J.Phys. D: Appl. Phys. 28, pp.2181-2187, (1995).

7. V. I. Konov, P. I. Nickitin, A. M. Prohorov, A. S. Silenok, Magnetic fields and currents generation at optical discharge in the recombining plasmas // Pisma v ZETF , 39(11), pp.837-842 (in Russian).

8. V. S. Burakov, N. V. Tarasenko, N. A. Tcheptsova, Investigating of laser plasma with resonant fluorescence technique // Zhurnal prikladnoi spekrtoskopii, 56(5-6), pp.837-842, (1992) (in Russian). 